\title{
Massenscreening und Einzeltherapie
}

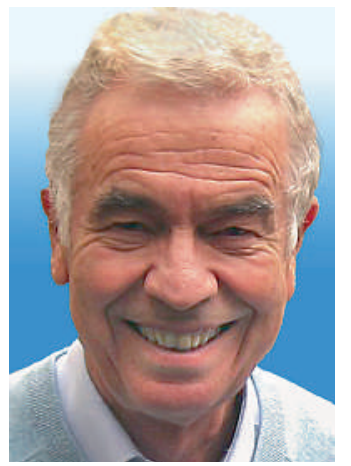

Hans Stalder
In der Nummer 48/2011 der Schweizerischen Ärztezeitung [1] gab es eine Empfehlung des Swiss Medical Board (SMB) zum Thema PSA (prostataspezifisches Antigen), deren Fazit - der PSA-Test eigne sich nicht zur Früherkennung des Prostatakarzinoms und solle nicht von den Kassen erstattet werden - von der Schweizerischen Gesellschaft für Urologie bestritten wird $[2,3]$. In dieser Ausgabe finden Sie Reaktionen unserer Leser und eine Antwort des Swiss Medical Board (ab Seite 80).

Es ist nicht neu, dass Fachverbände oder Ärzte eine andere Haltung zu Vorschriften zur Früherkennung oder zu Richtlinien eines neutralen, als unabhängig eingestuften Organs einnehmen. Dafür gibt es bestimmt andere Gründe als nur rein finanzielle Erwägungen, wie auf den ersten Blick angenommen werden könnte (obwohl wir nicht vergessen sollten, dass die U.S. Preventive Services Task Force (U.S.PSTF) wegen der mächtigen Lobby der Radiologen lange keine Richtlinie zur Prävention von Dickdarmkrebs durch Endoskopie veröffentlichen konnte ...).

Häufig vergessen die Ärzte, dass sich ihr individueller Therapieansatz von dem einer Instanz wie dem SMB oder der U.S.PSTF unterscheidet. Diese Instanzen basieren ihre Schlussfolgerungen auf Bevölkerungsstudien und müssen die Gesamtmortalität berücksichtigen, im Gegensatz zum Spezialisten, der sich mit einem bestimmten Patienten bzw. mit einer bestimmten Krankheit auseinandersetzt. Im Übrigen gelten ja die aus epidemiologischen Untersuchungen der Bevölkerung abgeleiteten Empfehlungen nicht unbedingt für den spezifischen Einzelpatienten.

Eine gute Früherkennung stützt sich auf mehrere Kriterien: 1. Die betreffende Krankheit muss ein wichtiges Gesundheitsproblem sein. 2. Der Test muss einfach sein. 3. Er muss hochempfindlich und spezifisch sein. 4. Die Früherkennung soll wenig Nebenwirkungen haben und 5. muss sie sich deutlich auf Morbidität und Mortalität auswirken. Der PSA-Test erfüllt ganz offenkundig nur Punkt 1 und 2 mit absoluter Sicherheit.

Es ist vor allem die mangelnde Spezifität, die Probleme bereitet, denn sie wirft eine ethische Frage auf: Ist es zulässig, Menschen Schaden zuzufügen, d. h., unnötig zu operieren und dabei erhebliche Nebenwirkungen zu provozieren, um anderen zu nutzen? Jedenfalls muss das Verhältnis zwischen dem Schaden für den einen und dem Nutzen für den anderen sehr niedrig sein. Offenkundig ist dies bei der Früher- kennung von Prostatakrebs nicht der Fall, ganz unabhängig von den variierenden Zahlen, die in der Literatur zu Nebenwirkungen der Untersuchungen und den Komplikationen einer unnötigen Prostatektomie zu finden sind. Ganz generell gibt es in der Früherkennung andere ethische Kriterien als bei der Therapie. Über eine hochriskante, für den Kranken jedoch notwendige Therapie zu entscheiden, ist etwas anderes, als eine Früherkennung für eine gesunde Person zu verordnen, die potentielle Risiken birgt. Das SMB richtet sich an Gesunde.

Das Pro und Kontra eines Medical Board und seiner Zusammensetzung sowie das Für und Wider eines Health Technology Assessments (HTA) wurden bei einer von der Schweizerischen Ärztezeitung organisierten Podiumsdiskussion in Zürich (s. Seite 56 dieser

\section{«Ganz generell gibt es in der Früherkennung andere ethische Kriterien als bei der Therapie.»}

Ausgabe) diskutiert. Ich persönlich meine, dass wir eine solche unabhängige Instanz brauchen, die sich sine ira et studio mit kontroversen medizinischen Themen auseinandersetzt. Das SMB war bewundernswerterweise mutig, das Thema PSA anzugehen, selbst wenn man sich fragen kann, ob dies politisch klug war. Ich jedenfalls freue mich bereits auf die Empfehlungen, die das Board zur Früherkennung von Brustkrebs durch die Mammographie herausgeben wird. Da müssen sie nämlich mit noch mächtigeren Lobbys rechnen ...

Hans Stalder*

\section{Literatur}

1 Swiss Medical Board. PSA-Test nicht geeignet zur Früherkennung des Prostatakarzinoms. Schweiz Ärztezeitung. 2011;92(48):1857.

2 Schweizerische Gesellschaft für Urologie. Weiterhin besonnene Anwendung des PSA-Tests empfohlen. Schweiz Ärztezeitung. 2011;92(49):1915.

3 Recker F. Swiss Medical Board: «das Kind mit dem Bade ausschütten». Schweiz Ärztezeitung. 2011;92(51/52):2002-4. 